\title{
Fibular (Peroneal) Nerve Entrapment Neuropathy-Review
}

\author{
Marcelo José da Silva Magalhães ${ }^{1 *}$, Gabriella Dias Falcão², Hérica Mendes Paiva ${ }^{2}$, Juliana Bispo Cunha², Marcelo \\ Rodrigo Martins de Oliveira ${ }^{2}$, Rafael Fagundes de Figueiredo ${ }^{2}$, Raíssa Santos Neves ${ }^{2}$, Victor Henrique Versiane \\ Alexandria ${ }^{2}$ and Henrique Nunes Pereira Oliva ${ }^{3}$
}

${ }^{1}$ Neurosurgery Department, Vila da Serra Hospital, Brazil

${ }^{2}$ Medicine student, Faculdades Unidas do Norte de Minas (FUNORTE), Brazil

${ }^{3}$ Medicine student, Faculdade de Medicina Integrada de Montes Claros (FipMOC), Brazil

*Corresponding author: Marcelo José da Silva de Magalhães, Capelinha Street, n 375, Sumaré- Montes Claros-Minas Gerais, Brazil Submission: March 16, 2018; Published: July 05, 2018

\begin{abstract}
Fibular nerve entrapment neuropathy (FNEN) is defined as involvement of the nerve in all its course. As etiology the idiopathic compressive form is the most common, but the traumatic form has a worse prognosis. Epidemiological data regarding FNEN are scarce due to lack of medical familiarity with the pathology in question. The diagnosis is clinical, and examinations of electroneuromyography of the lower limbs become useful for surgical and prognostic purposes. General and rehabilitative measures are instituted initially, but with little efficacy, surgery is indicated in the presence of a slowly progressive fibular neuropathy associated with extrinsic lesions or when the initial deficit is already severe. The prognosis varies with the etiology and the time between the onset of symptoms and the surgical approach. This study is a bibliographical review based on the specialized literature, through research of scientific articles selected in searches in the PubMed database
\end{abstract}

Keywords: Fibular nerve; Peroneal neuropathies; Nerve compression syndromes; Surgical decompression

\section{Introduction}

Fibular nerve entrapment neuropathy (FNEN) is a cause of numbness and pain in the lower thigh and dorsum of the foot and motor weakness of the extensors of the toes and ankle. It was first described by Henry in 1945. Neuropathy of the fibular nerve is considered as the most common compressive syndrome in the lower limbs and the third most frequent focal neuropathy cause[1-3].

FNEN is more common in men than in women at a 3.9: 1 ratio, the most common site of compression is the prominence of the fibular neck. Several predisposing factors are responsible for triggering FNEN predominating the idiopathic cause, in addition to other etiologies such as traumatic, lipomas, long periods of bed restriction, and chronic lateral compartment syndrome post fasciotomy[2-4].

Immediate evaluation of FNEN is essential because delayed diagnosis and treatment is associated with poorer prognosis in the long term and may be underdiagnosed due to the lack of familiarity of many clinicians with common causes and signs of compression [5].
This study aims to evaluate the prognostic factors based on the time between the onset of symptoms and the surgical approach.

\section{Methodology}

This study is a bibliographical review based on the specialized literature, through research of scientific articles selected in searches in the PubMed database. The descriptors "fibular nerve", "nerve entrapment" and "surgery" were used. There were included only articles whose patients had compressive disease of the fibular nerve and who underwent surgical treatment. Articles that were not provide effective data on the treatment were excluded.

\section{Discussion}

\section{Anatomy}

The common fibular nerve is formed by the posterior divisions of the spinal nerves L4, L5, S1 and S2. It originates in the bifurcation of the sciatic nerve, which occurs most often at the junction of the medial and distal thirds of the thigh. After its origin, the nerve directs obliquely in a lateral direction and, in the popliteal fossa, 
follows along the medial margin of the biceps femoris muscle. Adjacent to the head of the fibula, the nerve surrounds its cervix, later covered only by skin and subcutaneous tissue, and it courses towards the anterior compartment of the leg[4,6].

\section{Physiopathology}

In assessing patients with peripheral nerve disorders, the clinician must take into account the structural composition of the nerve and the pathophysiology of the nerve injury. The nerve fibers are enveloped in a layer of endoneurium, and are thus grouped into fascicles covered by perineurium, which in turn combine to form the peripheral nerves, which are covered by two layers of epineurium. Nerve dysfunction related to acute compression is caused by ischemic changes that can cause conduction blockage. Several degrees of endoneural edema are observed, but this usually resolves after approaching the causal factor. In chronic compression (greater than 28 days), conduction deceleration is observed along with an endoneuralinflammatory response. This response results in the formation of fibrous tissue may further exacerbate compression. Axonal degeneration is observed with compressions greater than 4 weeks[7].

\section{Clinical condition}

The motor deficit predominates in the initial phase manifesting itself by restriction of the extension of the toes, of the dorsal flexion and eversion of the ankle that determines the drop foot. Subjective sensory symptoms such as paresthesia and, more rarely, pain can be found on the dorsum of the foot, lateral side of the leg. Reflexes are generally preserved. A positive Tinel Signal can be found in the region of the head of the fibula. Palpation may reveal thickening of the nerve[3,4,7,8].

\section{Diagnosis}

Clinical tests can be performed with the patient at rest and after exercise, with the following tests: Pressure applied on the anterior intermuscular septum while the patient actively performs ankle dorsiflexion. In the second, it was done, plantar flexion, passively, and inversion in the ankle. Finally, while maintaining passive stretching, gentle percussion was applied along the nerve. Pain or paresthesia caused by any of these tests suggested fibular nerve compression.

Electromyoneurography of the lower limb is indicated to assist the clinician in identifying the location and severity of the nerve injury of the fibular nerve. The results of Electromyoneurography may help dictate the course of treatment. However, findings with no abnormalities do not exclude the diagnosis. When these findings suggest a more advanced disease, surgical intervention may be indicated and may increase the likelihood of a favorable outcome[7-9].Magnetic resonance imaging may help when suspicion is a compressive or tumoral cause [15-17].

Among the main diagnostic differences of the lesion of the fibular nerve is the lumbar disc herniation affecting the spinal nerve L5 (radiculopathy of L5), acting as a compressive lesion in the fibular segment of the sciatic nerve[15-17].
Polyneuropathy is another differential diagnosis defined as a simultaneous disorder in several peripheral nerves, which may include the fibular nerve. In polyneuropathy the clinical picture may be chronic with progressive development of symptoms. There may be burning pain, paresthesias, painful sensation that intensifies at night and worsens when the sensitive area is manipulated[15-17]. Besides these, the compression of the fibular nerve in the knee is another important differential diagnosis, the cause often not known, being denominated of idiopathic. Among the known causes of compression is the habit of crossing the legs, compression against bed grating in coma / debilitated patients, compression against surgical table, very tight rigid immobilizations, prolonged squatting position. Excessive weight loss may be a contributing factor to compression by the loss of the fatty cushion on the head of the fibula, which predisposes the nerve to external compression at this location[15-17].

Another important differential diagnosis is made with fibular nerve tumors. Tumors involving nerves can be divided into those arising from the nerve sheath, which are more frequent, and tumors that do not originate in the nerve sheath. Tumors originating from the nerve sheath may be benign (schwannomas, solitary neurofibromas and neurofibromas associated with Von Recklinghausen's disease) or malignant (malignant nerve sheath tumor). Tumors that do not originate from the sheath compromise the nerves through metastasis from a distant lesion or through direct extension from adjacent lesions[15-17].

\section{Treatment}

Therapy and use of devices may be useful for rehabilitation. Drop foot patients may use orthoses to improve walking. Physiotherapy should initially focus on stretching the contralateral muscle, including the plantar foot.

Patients with common fibular nerve compression should be observed for 2 to 4 months, time required to establish spontaneous recovery, should undergo clinical and sequentialelectromyoneurography. In general, the partial lesions present a faster recovery, when compared to the complete ones.

Surgical treatment is indicated when there is no recovery during the aforementioned observation period, even if the patient has only a sensory deficit. Surgery is also indicated in the presence of a slowly progressive fibular neuropathy associated with extrinsic lesions or when the initial deficit is already severe.

The surgery:Surgical approach to the common fibular nerve in the region of the head of the fibula is performed with the patient in the ventral decubitus, with the lower limb in extension or in the dorsal decubitus position with the lower limb with the flexed knee (Figure 1). The incision begins in the lateral region of the distal third of the thigh on its dorsal face, medial and parallel to the short head and tendon of the biceps femoris muscle. In a distal direction, it crosses the popliteal fold and proceeds to the posterior surface of the fibula head. From this point, the incision follows in a lateral direction, crossing the neck of the fibula, and is directed anteriorly to the anterior compartment of the leg between the tibialis anterior 
and extensor digitorum muscles. The incision should be made with caution on the lateral side of the fibula neck, since the common fibular nerve is superficial in this region. The dissection is initiated proximally, exposing the popliteal fascia, which is incised medial to the femoral biceps muscle [1-14].

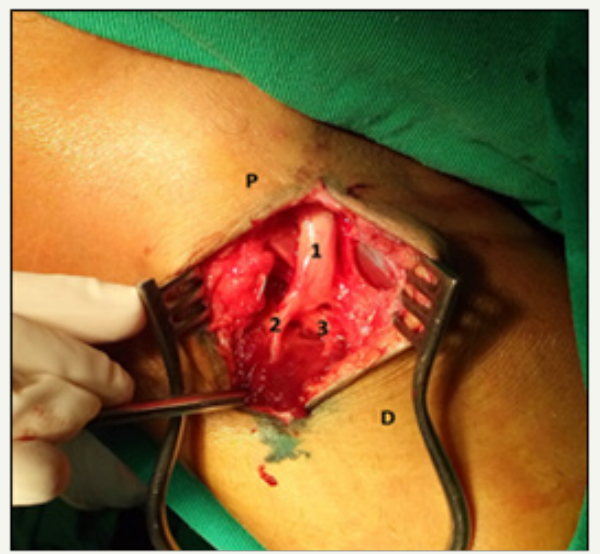

Figure 1: Per operative picture of surgical access to the common fibular nerve in the region of the head of the fibula.

P: Proximal to knee

D: Distal to knee

1: Common fibular nerve

2: Superficial fibular nerve

3: Deep fibular nerve

\section{Surgical Results}

Five studies were analyzed, totaling 75 patients, of which 59 $(78.67 \%)$ had satisfactory results and 16 classified the result as unchanged (21.32\%), according to Fisher's classification, and the British Medical Research Council (MRC). These patients were followed for about 40 weeks.

\section{Conclusion}

For patients with FNEN, spontaneous recovery is expected. However, it has been described as slow and / or incomplete. In the observed studies, a better prognosis was obtained for those patients who were treated early. The time to recovery in patients undergoing surgical treatment was lower than previously reported with conservative treatment. In addition, we observed that FNEN of traumatic origin has a worse prognosis.

\section{References}

1. Morimoto D, Isu T, Kim K, Sugawara A, Yamazaki K, et al. (2015) Microsurgical decompression for peroneal nerve entrapment neuropathy. Neurol Med Chir (Tokyo) 55(8): 669-673.
2. Styf J (1989) Entrapment of the superficial peroneal nerve: diagnosis and treatment by decompression - J Bone Joint Surg [Br] 71(1): 131-135.

3. Siqueira MG, Martins RS (2006) Síndromes Compressivas de Nervos periféricos-diagnóstico e tratamento. ( $1^{\text {st }}$ edn), Di Livros, Rio de Janeiro, Brazil.

4. Maalla R, Youssef M, Ben lassoued N, Sebai MA, Essadam H (2013) Peroneal nerve entrapment at the fibular head: Outcomes of neurolysis. Orthopaedics \& Traumatology: Surgery \& Research 99: 719-722.

5. Myers RJ, Murdock EE, Farooqi M, Van Ness G, Crawford DC (2015) A Unique Case of Common Peroneal Nerve Entrapment. Orthopedics 38(7): e644-e646.

6. Siqueira MG, Martins RS (2006) Anatomia cirúrgica das vias de acesso aos nervos periféricos. (1st edn), Di Livros, Rio de Janeiro, Brazil.

7. Poage C, Roth C, Scott B (2016) Peroneal nerve Palsy: evaluation and management - J Am Acad Orthop Surg 24(1): 1-10.

8. Styf J, Morberg P (1997) The superficial peroneal tunnel syndrome: results of treatment by decompression. J Bone Joint Surg [Br] 79(5): 801-803.

9. Grant TH, Omar IM, Dumanian GA, Pomeranz CB, Lewis VA (2015) Sonographic evaluation of common peroneal neuropathy in patients with foot drop. J Ultrasound Med 34(4): 705-711.

10. Titolo P, Panero B, Ciclamini D, Battiston B, Tos P (2013) New tendon transfer for correction of drop-foot in common peroneal nerve palsy. Clin Orthop Relat Res 471(10): 3382.

11. Iwamoto N, Isu T, Chiba Y, Kim K, Morimoto D, Yamazaki K, Isobe M (2015) Clinical feathers and treatment of peroneal nerve entrapment neuropathy. No Shinkei Geka 43(4): 309-316.

12. Aki Fukuda, Akinobu Nishimura, Shigeto Nakazora, Ko Kato, Akihiro Sudo (2016) Entrapment of Common Peroneal Nerve by Surgical Suture following Distal Biceps Femoris Tendon Repair. Case Rep Orthop.

13. Tompkins RP, Melling CW, Wilson TD, Bates BD, Shoemaker JK (1985) Arrangement of sympathetic fibers within the human common peroneal nerve: implications for microneurography. J Appl Physiol 115(10): 1553-1561.

14. Schiefer MA, Freeberg M, Pinault GJ, Anderson J, Hoyen H, et al. (2013) Selective activation of the human tibial and common peroneal nerves with a flat interface nerve electrode. J Neural Eng 10(5): 056006.

15. Hughes RAC (2002) Peripheral neuropathy. BMJ 324: 466-469.

16. Robinson L (2000) Traumatic injury to peripheral nerves. Muscle Nerve 23(6): 863-873.

17. de Schepper AM, Vanhoenacker FM, Parizel PM, Gielen JL (2005) Imaging of Soft Tissue Tumors. ( $3^{\text {rd }}$ edn), Springer-Verlag, Berlin. 
Creative Commons Attribution 4.0 International License

For possible submissions Click Here

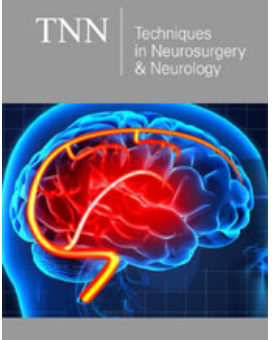

Techniques in Neurosurgery \& Neurology

\section{Benefits of Publishing with us}

- High-level peer review and editorial services

- Freely accessible online immediately upon publication

- Authors retain the copyright to their work

- Licensing it under a Creative Commons license

- Visibility through different online platforms 